\title{
Infection Control Practices \& Policies for Nursing Professionals
}

\section{POLICY - HAND HYGIENE}

\section{POINTS TO REMEMBER}

Learn how to clean your hands effectively

Wash your hands with soap and water:

- When starting a shift

- When visibly soiled

- Before drug rounds

- When serving food

\section{INTRODUCTION}

Hand hygiene is the single most important way of reducing cross-infection in the hospitals.

The purpose

1. To remove microbial contamination that has occurred by recent contact with patient / environment.

2. To prevent cross infection.

\section{The general guidelines}

- During clinical work, wear sleeves above the elbows.

- DO NOT WEAR a wristwatch or costume jewellery.

Hand washing should be carried out using either liquid soap (social hand washing) or using an approved skin disinfectant before a clinical procedure.

Alcohol rub (Sterilium) is to be used in high dependency areas as an alternative to hand washing to reduce bacterial load on clean hands.
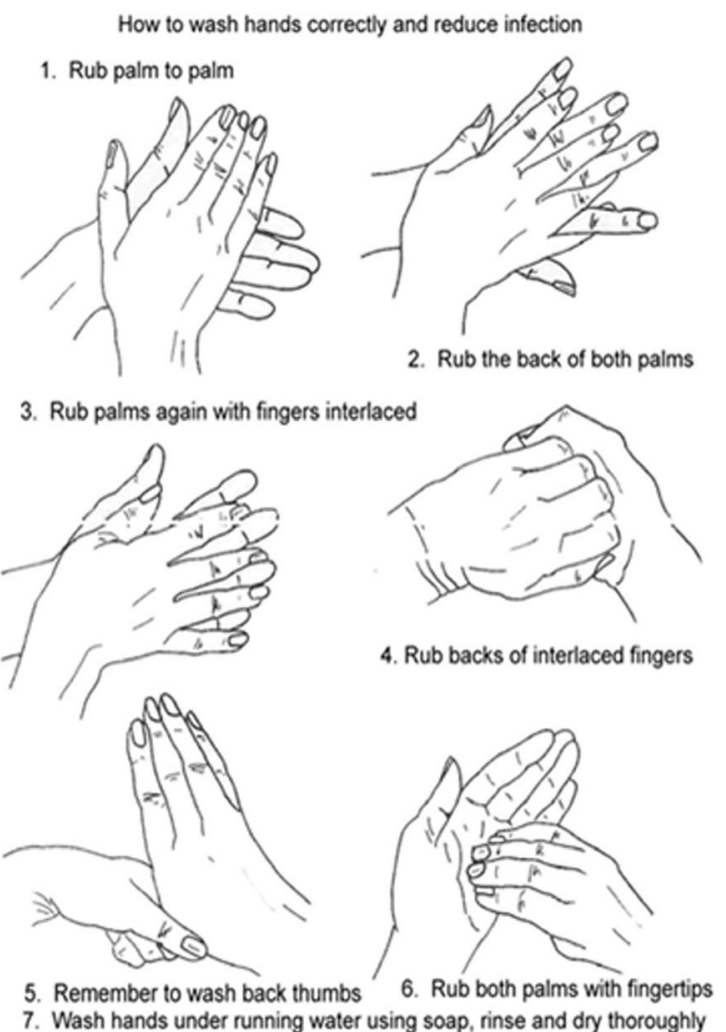